Chemical Physics 10 (1975) 185-198

(c) North-Holland Publishing Company

\title{
ON THE APPLICATION OF GROUP THEORY TO MOLECULAR EXCTTONS
}

\author{
Joseph HOSHEN * and Raoul KOPELMAN ${ }^{*}$ \\ and \\ Joshua JORTNER \\ Department of Chemistry. Tel-Aviv University, Tel-Aviv, Israel
}

Department of Chemistry. The University of Michigan, Ann Arbor, Michigan 48104, USA

Received 6 January 1975

\begin{abstract}
In this paper a group theoretical approach was employed for the classification and construction of molecular exciton wavefunctions for two important cry stal structures (naphthalene-anthracene and benzene), utilizing the representation theory of finite groups. The generally valid scheme requires only cyclic boundary conditions (being explicitly iniposed on all space group operations, including rotations and reflections). Even though these symmetry considerations are insufficient to determine crystal wavefunctions belonging to a general $k$ vector, it is still possible to write a simple expression for such wavefunctions. This is achieved for cases where the nonvanishing exciton transfer integrals are confined to molecular interactions along symmetry axes and/or planes.
\end{abstract}

\section{Introduction}

Ever since the pioneering studies of Davydov [1a], and of Winston and Halford [1b] on molecular excitons and phonons, space group symmetry has been extensively utilized for the determination of the band structure and optical properties of molecular crystals [2]. Most of the work has been concentrated on the "optically active" states for which $k=0$. In recent years more interest has been devoted to the study of the entire exciton band [3]. Experimental results from hot band spectroscopy furnished information on the density of states for the entire exciton band [4]. Several investigations [5] were aimed towards the study of substitutionally disordered crystals where the $\boldsymbol{k}$ selection rule for the optical transition is not valid, so that some limited information on the entire electronic band can also be obtained. On the other hand, the disordered crystal solutions are obtained from zeroth-order perfect crystal functions, thus retaining the importance of symmetry classifications.

It would be useful to obtain a simple method to classify and construct crystal wavefunctions according to the irreducible representations of the crystal space group for the various $k$ wavevectors. Another interesting question concerns the conditions for a simple representation of the wavefunctions belonging to a general $k$ vector. It is known that crystal wavefunctions belonging to special $k$ vectors can be determined by symmetry [2a]. It is an interesting question in this context to determine the conditions under which wavefunctions can be compared to functions belonging to special $k$ vectors. The simplification of wavefunctions belonging to general $k$ vectors is relevant to the utilization of the experimental results of hot band spectroscopy [4], and for the determination of the density of exciton states in the pure crystal. In addition, Green's function matrix elements tend to be very complex $[6,5 c]$ if the crystal wavefunctions for a gereral $k$ cannot be simplified.

\footnotetext{
* Supported by NSF Grant GH-32578X.

F Partially supported by NIH Grant NS08116 and partially supported by NIH Special Research Fellowship 1-F03 GMS3602-0L at Tel-Aviv University.
} 
In the next section a general scheme will be presented for the classification of crystal states and the construction of crystal wavefunctions, utilizing the representation theory of finite groups. In section 3 the crystal states of the two important cases, naphthalene $\left(C_{2 h}^{5}\right)$ and benzene $\left(D_{2 h}^{15}\right)$ will be investigated. These crystals contain two and four molecules per unit cell, respectively, where all molecules are interchange equivalent. Section 4 is devoted to the discussion of wavefunctions belonging to a general $k$ vector.

\section{The application of group theory for the determination of wavefunctions in molecular crystals}

In this section we consider a crystal wavefunction corresponding to a single molecular excited state $f$, and a crystal containing $\sigma_{D}$ molecules per unit cell. The crystal wavefunction is given by:

$$
|k, j\rangle=\sum_{\alpha=1}^{\sigma_{D}} B_{\alpha j}(k)\left|k, s_{\alpha}\right\rangle,
$$

where $s_{\alpha}$ is a vector denoting the location of the $\alpha$ molenule in the unit cell, $k$ is the wavevector, $j$ is the exciton branch index, and $\left|k, s_{\alpha}\right\rangle$ is the site excitation wavefunction. The excitation index $f$ is omitted since only a single molecular state is considered in this paper.

The site excitation wavefunction $\left|\boldsymbol{k}, s_{\boldsymbol{\alpha}}\right\rangle$ is represented by:

$$
\left.\left|k, s_{\alpha}\right\rangle=(1 / \sqrt{N}) \sum_{n} \exp \left(\mathrm{i} k-R_{n}\right) \mid R_{n}+s_{\alpha}\right),
$$

where $\left|R_{n}+s_{\alpha}\right\rangle$ denotes a localized excitation at a site $\alpha$ of the $n$th unit cell and $N$ is the number of unit cells in the crystal. $R_{n}$ is spanned by the three primitive vectors $a_{1}, a_{2}$ and $a_{3}$ :

$$
R_{n}=n_{1} a_{1}+n_{2} a_{2}+n_{3} a_{3}
$$

where $n_{i}=1,2, \ldots, N_{i}$, and $N_{i}$ is the number of unit cells in the $a_{i}$ direction, so that $N=N_{1} N_{2} N_{3}$. The wavevector $k$ can be represented in terms of the reciprocal primitive lattice vectors $b_{1}, b_{2}$, and $b_{3}$ :

$$
k=\mu_{1} b_{1}+\mu_{2} b_{2}+\mu_{3} b_{3}
$$

where $\mu_{i}$ satisfies the condition $-\frac{1}{2}<\mu_{i} \leqslant \frac{1}{2}$. The reciprocal lattice vectors are subjected to the following orthogonality relation:

$$
a_{i} \cdot b_{j}=2 \pi \delta_{i j}
$$

A cyclic boundary condition is imposed on the crystal. In addition it is assumed that $N_{1}, N_{2}$, and $N_{3}$ are even numbers, so that the $k$ vector attains values for all special points, within, and on the boundary of the Brillouin zone for a finite $N$.

The coefficients $B_{\alpha j}(k)$ of eq. (1) can be determined by diagonalizing the crystal hamiltonian $\hat{H}$, corresponding to the excitation in question. Since $\left.\mid k, s_{\alpha}\right)$ are diagonal in $k$, it is necessary to diagonalize $\sigma_{D} \times \sigma_{D}$ matrix elements

$$
L_{\alpha \beta}(k)=\left\langle k, s_{\alpha}|\hat{H}| k, s_{\beta}\right\rangle \text {. }
$$

The crystal hamiltonian $\hat{H}$ commutes with all space group operations, hence, it is possible to construct symmetry wavefunctions belonging to irreducible representations of the space group.

In the next section we shall adopt the regular prescription [7] for the classification and construction of wavefunctions according to the irreducible representations of a finite group. This procedure is adequate for the treatment of infinite discrete (non-continuous) groups [8].

The procedure for the construction of the symmetry adapted wavefunctions is straightforward, and can be summarized in two steps: 
(a) - A reducible representation $\Gamma$ of the crystal is constructed. This is a $\sigma_{\mathrm{D}} N \times \sigma_{\mathrm{D}} N$ dimensional matrix, spanned by the localized excitation wavefunction $\left|R_{n}+s_{\alpha}\right\rangle$. Since we are dealing with a crystal where each molecule can be mapped onto the other by a spacegroup operation, each row of the representation matrix will be filled with zeros, except for one matrix element of that row. The nonzero element of the row has the absolute value of one, and corresponds to the transformation of $\left|R_{n}+s_{\alpha}\right\rangle$. The crystal wavefunctions can be classified according to the irreducible representations by utilizing the following formula:

$$
n_{\nu}=(1 / h) \sum_{R}\left[X^{(\nu)}(R)\right]^{*} x(R),
$$

where $n_{\nu}$ is the number of times the irreducible representation $D^{(v)}$ appears in the reducible representation $\Gamma$. $\chi^{(\nu)}(R)$ and $\chi(R)$ are the traces of $D^{(\nu)}$ and $\Gamma$, respectively, corresponding to the element $R$ of the group.

There are two immediate results for $\Gamma$ :

(1) The trace of the identity operation $\{\epsilon \mid \theta\}$ is $\sigma_{D} N$, since all localized excitations are invariant under the identity operation.

(2) The trace of the pure translation subgroup $\left\{\epsilon \mid \tau_{n}\right\}$ and operations involving screw axes and glide planies $\left\{\delta \mid \alpha(\hat{O})+\tau_{n}\right\}$ is zero.

(b) - Wavefunctions belonging to the row $i$ of the irreducible representation $D^{(v)}$ are given by [7]

$$
\psi(\nu)=\left(l_{\nu} / h\right) \sum_{R}\left[D^{(\nu)}(R)\right]_{i i}^{*} \hat{o}_{R} \psi,
$$

where $\hat{O}_{R}$ is a symmetry operator, corresponding to the group element $R, \psi_{i}^{(\nu)}$ is a function belonging to the $i$ th row of the $\nu$ th irreducible representation of the group of dimension $l_{v}$.

\section{Application of representation theory to crystals containing two and four molecules per unit cell}

\subsection{Two molecules per unit cell: The naphthalene-anthracene $\left(\mathrm{C}_{2 h}^{5}\right)$ space group}

The symmetry elements of crystals belonging to the naphthalene-anthracene $C_{2 h}^{5}$ space group irclude a twofold screw axis $C_{2}$ parallel to the $a_{3}$ lattice vector and a glide plane $\sigma$ perpendicular to the screw axis. A molecule is located at a lattice site $\tau / 2$, where $\tau=\left(a_{1}+a_{3}\right) / 2$. All other molecules are related to this molecule by the $C_{2 h}^{5}$ space group operations. The inversion centers of the molecules in the crystal are located at lattice sites $\pm \tau / 2+R_{n}$.

The four basic symmetry operations are: $\{\epsilon \mid 0\} ;\left\{C_{2} \mid\left(a_{3} / 2\right)\right\} ;\{I \mid \tau\} ;\left\{\sigma \mid\left(a_{1} / 2\right)\right\}$. The remaining elements of the space group can be generated by multiplication of the pure translation operation $\left\{\epsilon \mid R_{n}\right\}$ by the four basic operations.

The trace of the reducible representation spanned by the localized excitation will be non-zero for the identity operation and for some of the inversion operators (see appendix). Assuming that the molecular wavefunctions are ungerade, and therefore change sign under an inversion operation, the trace of $\Gamma\left(\left\{I \mid R_{n}+\tau\right\}\right)$ can be given explicitly by the following compact form:

$$
\chi\left(\left\{I \mid R_{n}+\tau\right\}\right)=-2\left[1+(-1)^{n_{2}}\right]\left[1+(-1)^{n_{1}+n_{3}}\right] .
$$

The trace of the identity element $\{\epsilon \mid 0\}$ is $2 N$. Utilizing eq. (7), the representation $\Gamma$ is readily reduced into its irreducible components. The result is displayed [9] in table 1 . The traces of the irreducible representation of table 1 are determined from table 2.

It is apparent from table 1 that it is necessary to diagonalize a $2 \times 2$ hamiltonian matrix only for a general $k$, $i$.e. $k_{15}$. In all other cases it is possible to choose the basic wavefunctions invoking symmetry considerations only. 
Table 1

Reduction of the $\Gamma$ reducible representation into its irreducible components $D_{k_{j}}^{(\nu)}$ for $C_{2 h}^{S}$

\begin{tabular}{|c|c|c|c|c|c|}
\hline & Wave vector $k_{j}$ & $\nu$ & $x(\{e \mid 0\})$ & $x\left(\left\{I \mid R_{n}+r\right\}\right)$ & $n_{k_{i}}^{(\nu)}$ \\
\hline \multirow{33}{*}{ · } & $k_{7}=0$ & 1 & 1 & 1 & $\mathbf{0}$ \\
\hline & $k_{7}=0$ & 2 & 1 & -1 & 1 \\
\hline & $k_{7}=0$ & 3 & 1 & 1 & $\mathbf{0}$ \\
\hline & $k_{7}=0$ & 4 & 1 & -1 & 1 \\
\hline & $k_{13}=\frac{1}{2} b_{2}$ & 1 & 1 & $(-1)^{n_{2}}$ & 0 \\
\hline & $k_{13}=\frac{T}{2} b_{2}$ & 2 & 1 & $-(-1)^{n_{2}}$ & 1 \\
\hline & $k_{13}=\frac{\pi}{2} b_{2}$ & 3 & 1 & $(-1)^{n_{2}}$ & 0 \\
\hline & $k_{13}=\frac{\bar{l}}{2} b_{2}$ & 4 & 1 & $-(-1)^{n_{2}}$ & 1 \\
\hline & $k_{8}=\frac{1}{2}\left(b_{1}+b_{3}\right)$ & 1 & 1 & $-(-1)^{n_{1}+n_{3}}$ & 1 \\
\hline & $k_{8}=\frac{1}{2}\left(b_{1}+b_{3}\right)$ & 2 & 1 & $(-1)^{n_{1}+n_{3}}$ & $\mathbf{0}$ \\
\hline & $k_{8}=\frac{1}{2}\left(b_{1}+b_{3}\right)$ & 3 & 1 & $-(-1)^{n_{1}+n_{3}}$ & 1 \\
\hline & $k_{8}=\frac{1}{2}\left(b_{1}+b_{3}\right)$ & 4 & 1 & $(-1)^{n_{1}+n_{3}}$ & 0 \\
\hline & $k_{10}=\frac{1}{2}\left(b_{1}+b_{2}+b_{3}\right)$ & 1 & 1 & $(-1)^{n_{1}+n_{2}+n_{3}}$ & 1 \\
\hline & $k_{10}=\frac{1}{2}\left(b_{1}+b_{2}+b_{3}\right)$ & 2 & 1 & $(-1)^{n_{1}+n_{2}+n_{3}}$ & $\mathbf{0}$ \\
\hline & $k_{10}=\frac{1}{2}\left(b_{1}+b_{2}+b_{3}\right)$ & 3 & 1 & $(-1)^{n_{1}+n_{2}+n_{3}}$ & 1 \\
\hline & $k_{10}=\frac{1}{2}\left(b_{1}+b_{2}+b_{3}\right)$ & 4 & 1 & $(-1)^{n_{1}+n_{2}+n_{3}}$ & 0 \\
\hline & $k_{12}=\frac{1}{2} b_{1}$ & 1 & 2 & 0 & 1 \\
\hline & $k_{14}=\frac{1}{2}\left(b_{1}+b_{2}\right)$ & 1 & 2 & 0 & 1 \\
\hline & $k_{11}=\frac{1}{2} b_{3}$ & 1 & 2 & 0 & 1 \\
\hline & $k_{9}=\frac{1}{2}\left(b_{2}+b_{3}\right)$ & 1 & 2 & 0 & 1 \\
\hline & $k_{1}=\mu_{1} b_{1}+\mu_{2} b_{2}$ & 1 & 2 & 0 & 1 \\
\hline & $k_{1}=\mu_{1} b_{1}+\mu_{2} b_{2}$ & 2 & 2 & 0 & 1 \\
\hline & $k_{2}=\mu_{1} b_{2}+\frac{1}{2} \mu_{2} b_{2}+\frac{1}{2} b:$ & 1 & 2 & 0 & 1 \\
\hline & $k_{2}=\mu_{1} b_{1}+\mu_{2} b_{2}+\frac{1}{2} b_{3}$ & 2 & 2 & 0 & 1 \\
\hline & $k_{3}=\mu_{3} b_{3}$ & 1 & 2 & 0 & 1 \\
\hline & $k_{3}=\mu_{3} b_{3}$ & 2 & 2 & 0 & 1 \\
\hline & $k_{5}=\mu_{3} b_{3}+\frac{1}{2} b_{2}$ & 1 & 2 & 0 & $\downarrow$ \\
\hline & $k_{5}=\mu_{3} b_{3}+\frac{1}{2} b_{2}$ & 2 & 2 & 0 & 1 \\
\hline & $k_{6}=\frac{1}{2}\left(b_{1}+b_{2}\right)+\mu_{3} b_{3}$ & 1 & 2 & 0 & 1 \\
\hline & $k_{6}=\frac{2}{2}\left(b_{1}+b_{3}\right)+\mu_{3} b_{3}$ & 2 & 2 & $\mathbf{0}$ & 1 \\
\hline & $k_{4}=\frac{1}{2} b_{1}+\mu_{3} b_{3}$ & 1 & 2 & 0 & 1 \\
\hline & $k_{4}=\frac{1}{2} b_{1}+\mu_{3} b_{3}$ & 2 & 2 & 0 & 1 \\
\hline & $k_{15}=\mu_{1} b_{1}+\mu_{2} b_{2}+\mu_{3} b_{3}$ & 1 & 4 & 0 & 2 \\
\hline
\end{tabular}

The symmetry adapted wavefunctions for $k_{1}, k_{2}, k_{3}, k_{4}, k_{5}$ and $k_{6}$ are displayed for the $\mathrm{D}_{k_{j}}^{(1)}$ representation

$$
\left.|k,+\rangle=(1 / \sqrt{2} N) \sum_{n} \exp \left(i k \cdot R_{n}\right)\left(\mid R_{n}-\tau / 2\right)+\exp (i k \cdot \tau)\left|R_{n}+\tau / 2\right\rangle\right) \text {. }
$$
while for $D_{k j}^{(2)}$

$$
|k,-\rangle=(1 / \sqrt{2} N) \sum_{n} \exp \left(\mathrm{i} k \cdot R_{n}\right)\left(\left|R_{n}-\tau / 2\right\rangle-\exp (\mathrm{i} k \cdot \tau)\left|R_{n}+\tau / 2\right\rangle\right)
$$




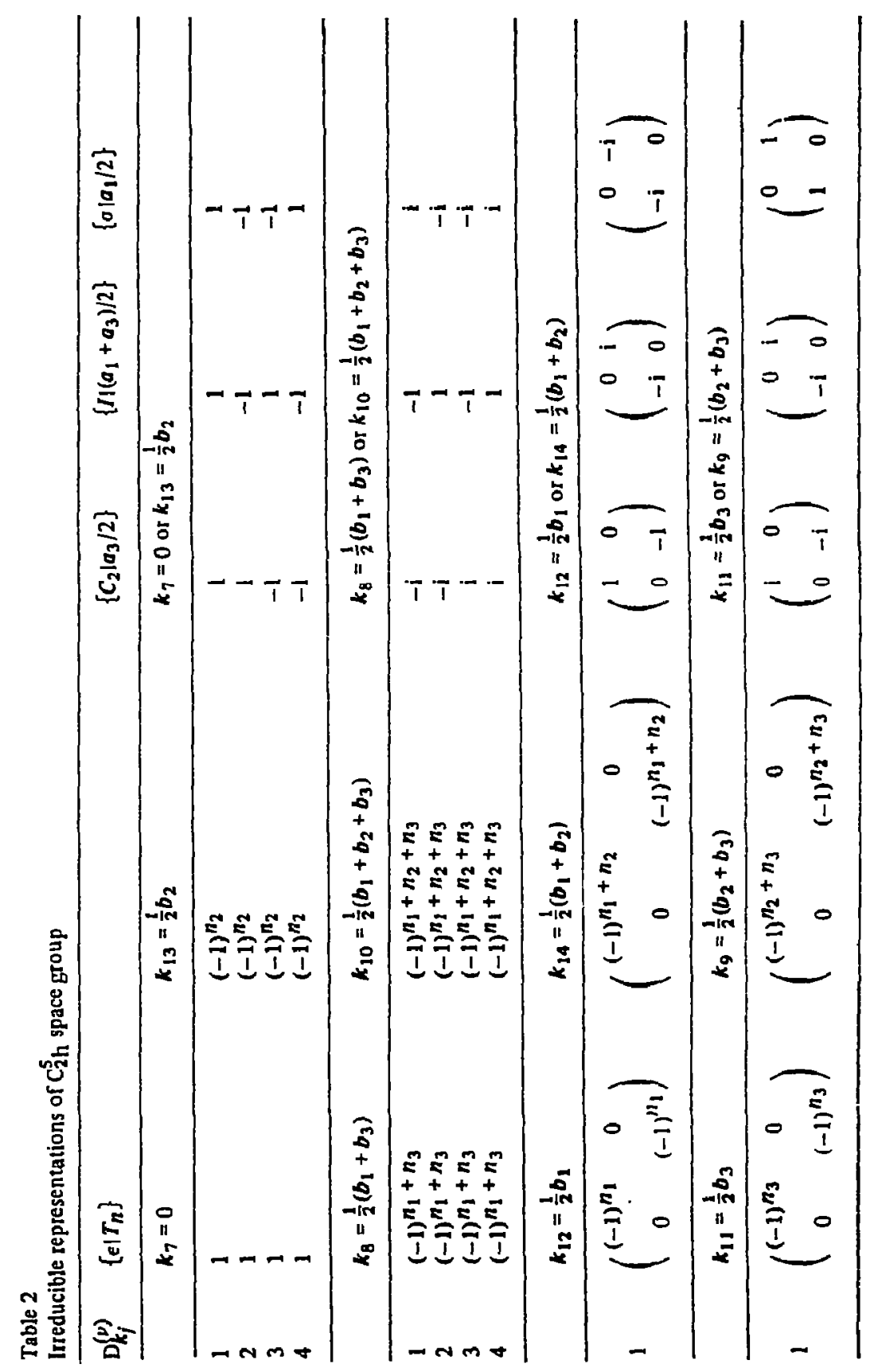




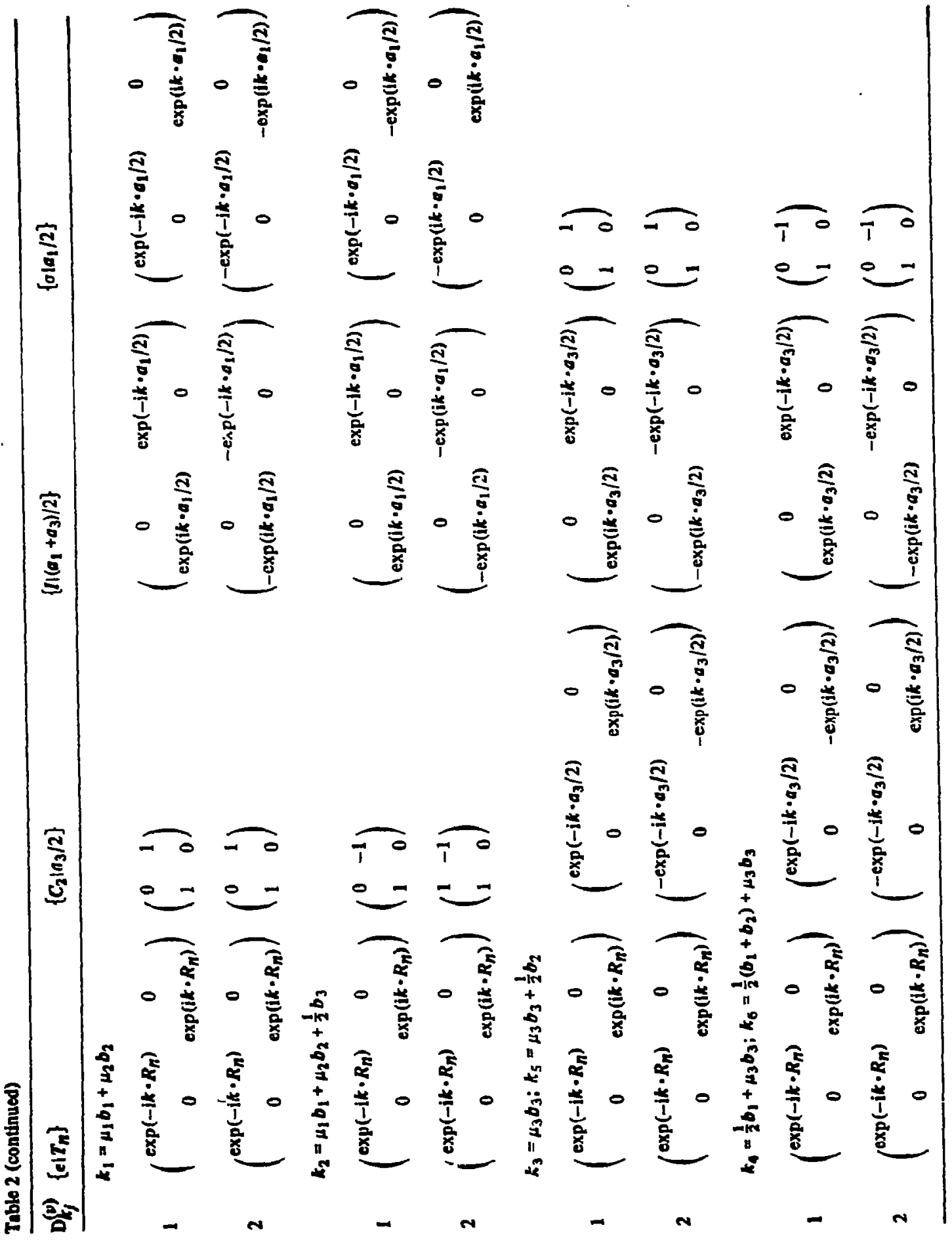




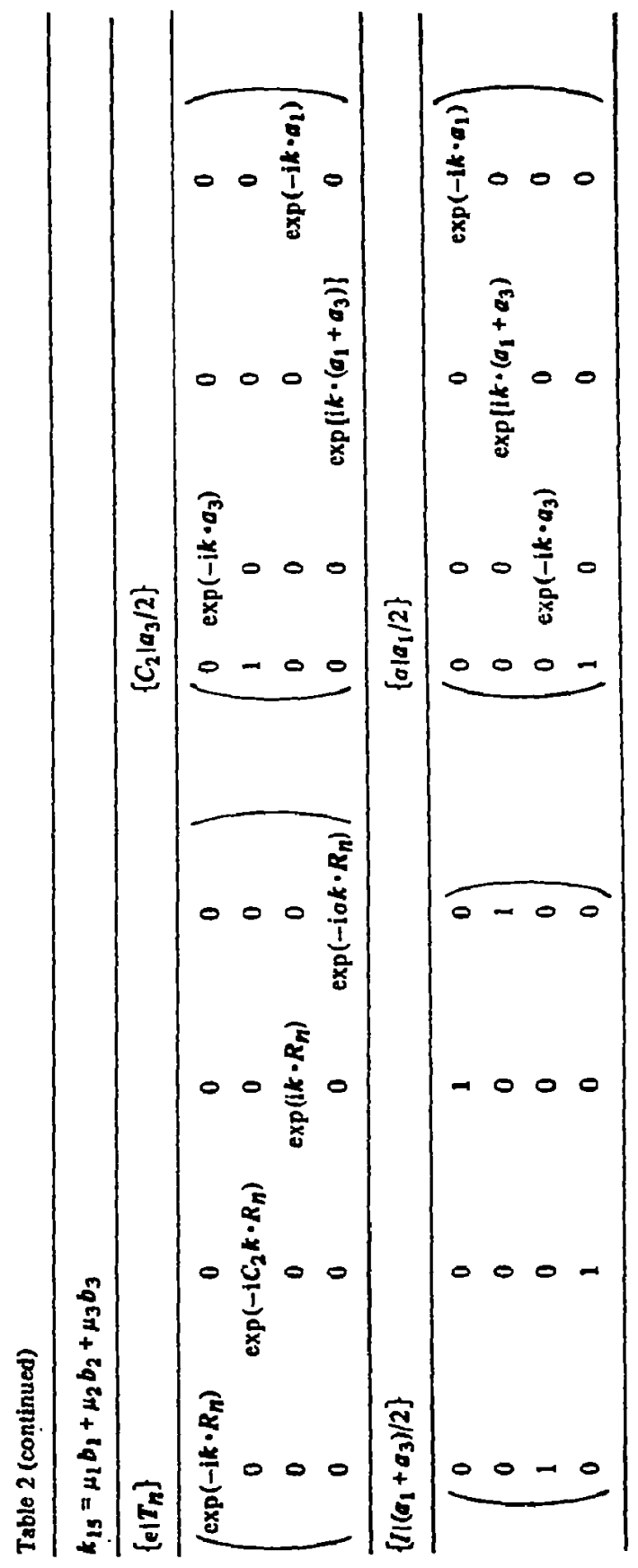


The partner functions of $|k,+\rangle$ and $|k,-\rangle$ are $|-k,+\rangle$ and $|-k,-\rangle$, respectively. This degeneracy does not exist for special points of the Brillouin zone $k_{7}, k_{8}, k_{10}, k_{13}$ (see table 1 ). However, $|k,+\rangle$ and $|k,-\rangle$ form a degenerate pair of partner functions on the Brillouin zone boundary for points $k_{9}, k_{11}, k_{12}$, and $k_{14}$.

\subsection{Four molecules per unit cell: The benzene space group $\mathrm{D}_{2 \mathrm{~h}}^{15}$}

The $D_{2 h}^{15}$ space group includes the eight basic operations

$$
\begin{array}{llll}
\{\epsilon \mid 0\} ; & \left\{c_{x} \mid \tau_{x}+\tau_{y}\right\} ; & \left\{c_{y} \mid \tau_{y}+\tau_{z}\right\} ; & \left\{c_{z} \mid \tau_{z}+\tau_{x}\right\} ; \\
\{I \mid 0\} ; & \left\{\sigma_{y z} \mid \tau_{x}+\tau_{y}\right\} ; & \left\{\sigma_{z x} \mid \tau_{y}+\tau_{z}\right\} ; & \left\{\sigma_{x y} \mid \tau_{z}+\tau_{x}\right\} .
\end{array}
$$

Since the three primitive vectors $a_{1}, a_{2}$ and $a_{3}$ are orthogonal, they coincide with the cartesian axes $x, y$, and $z$, respectively. $c_{i}$ denotes twofold rotation around the $i$ axis $(i=x, y, z), a_{i j}$ denotes mirror reflection by the $i j$ plane, and $\tau_{x}, \tau_{y}, \tau_{z}$ specific $a_{1} / 2, a_{2} / 2, a_{3} / 2$, respectively.

The localized excitations of the crystal can be generated by applying the first four operations of eq. (12) to. gether with a translation on the localized excitation 10 :

$$
\begin{aligned}
& \left|R_{n}\right\rangle=\delta\left(\left\{\epsilon \mid R_{n}\right\}\right)|0\rangle, \\
& \left|R_{n}+\tau_{x}+\tau_{y}\right\rangle=\delta\left(\left\{c_{x} \mid R_{n}+\tau_{x}+\tau_{y}\right\}\right)|0\rangle, \\
& \left|R_{n}+\tau_{y}+\tau_{z}\right\rangle=\hat{O}\left(\left\{c_{y} \mid R_{n}+\tau_{y}+\tau_{z}\right\}\right)|0\rangle, \\
& \left|R_{n}+\tau_{z}+\tau_{x}\right\rangle=\hat{O}\left(\left\{c_{z} \mid R_{n}+\tau_{z}+\tau_{x}\right\}\right)|0\rangle .
\end{aligned}
$$

These $4 N$ localized excitation wavefunctions form a basis for a $4 N$ dimensional reducible representation $\Gamma$. The only symmetry operations which fumishes a non-zero trace are the identity and some inversion operations. These traces are

$$
\begin{aligned}
& \chi(\{\epsilon \mid O\})=4 N, \\
& \chi\left(\left\{I \mid R_{n}\right\}\right)=-4 N\left[1+(-1)^{n_{1}+n_{2}+n_{3}}\right] .
\end{aligned}
$$

The reduction of $\boldsymbol{\Gamma}$ is accomplished by utilizing eq. (7). The results for the reduction of $\boldsymbol{\Gamma}$ are displayed in table 3. Since the three axes $x, y$, and $z$ are equivalent from a space group symmetry point of view, only results for one set of equivalent points is given.

The symmetry wavefunctions for $k_{7}$ and $k_{10}$ (see table 3 ) are determined by symmetry only, and so do their equivalent wavefunctions for $k$ parallel to the $y$ and $z$ axes. Utilizing eq. (8) and the representation table given by Kovalev [9], wavefunctions belonging to $k_{7}$ and $k_{10}$ (and their equivalent points) can be summed up:

$$
\begin{aligned}
& |k, 1\rangle=\frac{1}{2}\left\{|k, 0\rangle+\left|k, \tau_{x}+\tau_{y}\right\rangle+\left|k, \tau_{y}+\tau_{z}\right\rangle+\left|k, \tau_{z}+\tau_{x}\right\rangle\right\}, \\
& |k, 2\rangle=\frac{1}{2}\left\{|k, 0\rangle+\left|k, \tau_{x}+\tau_{y}\right\rangle-\left|k, \tau_{y}+\tau_{z}\right\rangle-\left|k, \tau_{z}+\tau_{x}\right\rangle\right\}, \\
& |k, 3\rangle=\frac{1}{2}\left\{|k, 0\rangle-\left|k, \tau_{x}+\tau_{y}\right\rangle+\left|k, \tau_{y}+\tau_{z}\right\rangle-\left|k, \tau_{z}+\tau_{x}\right\rangle\right\}, \\
& |k, 4\rangle=\frac{1}{2}\left\{|k, \theta\rangle-\left|k, \tau_{x}+\tau_{y}\right\rangle-\left|k, \tau_{y}+\tau_{z}\right\rangle+\left|k, \tau_{z}+\tau_{x}\right\rangle\right\},
\end{aligned}
$$

where

$$
\begin{aligned}
& |k, 0\rangle=N^{-1 / 2} \sum_{n} \exp \left(\mathrm{ik} \cdot R_{n}\right)|0\rangle, \\
& \left|k, \tau_{x}+\tau_{y}\right\rangle=N^{-1 / 2} \sum_{n} \exp \left[\mathrm{i} k \cdot\left(R_{n}+\tau_{x}+\tau_{y}\right)\right]\left|R_{n}+\tau_{x}+\tau_{y}\right\rangle,
\end{aligned}
$$


Table 3

The benzene crystal space group $D_{2 h}^{15}$

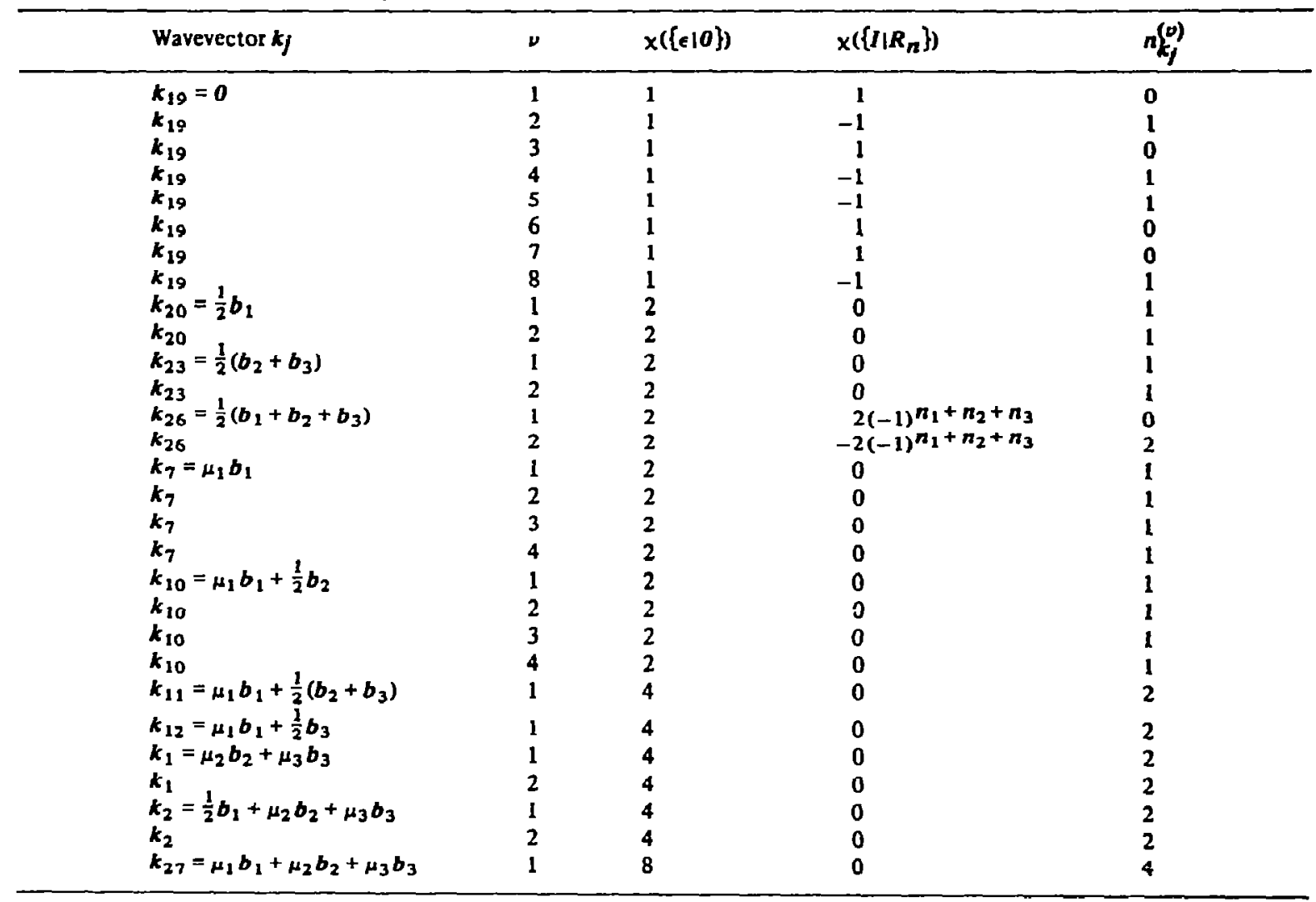

$\left|k, \tau_{y}+\tau_{z}\right\rangle=N^{-1 / 2} \sum_{n} \exp \left[\mathrm{i} k \cdot\left(R_{n}+\tau_{y}+\tau_{z}\right)\right]\left|R_{n}+\tau_{y}+\tau_{z}\right\rangle$,

$$
\left.\left|k, \tau_{z}+\tau_{x}\right\rangle=N^{-1 / 2} \sum_{n} \exp \left[\mathrm{i} k \cdot\left(R_{n}+\tau_{z}+\tau_{x}\right)\right] \mid R_{n}+\tau_{z}+\tau_{x}\right)
$$

Each function $|k, i\rangle$ has a partner $|-k, i\rangle$ except for the point $k=0$. Points $k_{20}, k_{23}$ (and their equivalent points), (see table 3 ) correspond to two sets of doubly degenerate wavefunctions. It should be noted that two wavefunctions belong to each row of the representations of $k_{1}, k_{2}, k_{11}, k_{12}$ and $k_{26}$, hence, it is necessary to diagonalize a $2 \times 2$ hamiltonian matrix for these wavefunctions. As anticipated, a $4 \times 4$ secular matrix has to be solved for the general $k$ vector $\left(k_{27}\right)$.

\section{Simplified solution for a general $k$ vector}

\subsection{Two molecules per unit cell}

The most convenient choice of a basis set of wavefunctions for a general $k$ vector for a crystal containing two 
molecules per unit cell, belonging to $C_{2 h}^{5}$ space group, is

$$
|k,-\tau / 2\rangle \text { and } \exp (\mathrm{i} k \cdot \tau)|k, \tau / 2\rangle \text {. }
$$

The exp(ik - r) factor ensures that the h.miltonian matrix is real-symmetric, provided that the localized excitations are real functions (see below). The $2 \times 2$ hamiltonian matrix is:

$$
\begin{aligned}
& H(k)=\left(\begin{array}{ll}
L_{11}(k) & L_{12}(k) \\
L_{21}(k) & L_{22}(k)
\end{array}\right), \\
& L_{11}(k)=\sum_{n} \exp \left(\mathrm{ik} \cdot R_{n}\right)\left\langle-\tau / 2|\hat{H}|-\tau / 2+R_{n}\right\rangle, \\
& L_{22}(k)=\sum_{n} \exp \left(\mathrm{i} k \cdot R_{n}\right)\left(\tau / 2|\hat{H}| \tau / 2+R_{n}\right\rangle, \\
& L_{12}(k)=\exp (\mathrm{i} k \cdot \tau) \sum_{n} \exp \left(\mathrm{i} k \cdot R_{n}\right)\left\langle-\tau / 2|\hat{H}| \tau / 2+R_{n}\right\rangle \\
& L_{21}(k)=\exp (-\mathrm{i} k \cdot \tau) \sum_{n} \exp \left(\mathrm{i} k \cdot R_{n}\right)\left(\tau / 2|\hat{H}|-\tau / 2+R_{n}\right\rangle .
\end{aligned}
$$

Applying the operation $\left\{J \mid \tau+R_{n}\right\}$ to each term in the summation of eq. (27d) we obtain:

$$
L_{12}(k)=\exp (i k \cdot \tau) \sum_{n} \exp \left(i k \cdot R_{n}\right)\left\langle 3 \tau / 2+R_{n}|\hat{H}| \tau / 2\right\rangle .
$$

Since we assumed the molecular wavefunctions to be real $L_{12}(k)$ can be recast in the form:

$$
\begin{aligned}
& L_{12}(k)=\exp (\mathrm{ik} \cdot \tau) \sum_{n} \exp \left(\mathrm{ik} \cdot \boldsymbol{R}_{n}\right)\left\langle\tau / 2|\hat{H}| 3 \tau / 2+\boldsymbol{R}_{n}\right\rangle \\
& \quad=\exp (\mathrm{ik} \cdot \tau) \exp (-\mathrm{i} k \cdot 2 \tau) \sum_{n} \exp \left[\mathrm{ik} \cdot\left(\boldsymbol{R}_{n}+2 \tau\right)\right]\left\langle\tau / 2|\hat{H}|-\tau / 2+\boldsymbol{R}_{n}+2 \tau\right\rangle \\
& \quad=\exp (-\mathrm{i} k \cdot \tau) \sum_{n} \exp \left(\mathrm{i} k \cdot \boldsymbol{K}_{n}\right)\left\langle\tau / 2|\hat{H}|-\tau / 2+\boldsymbol{R}_{n}\right\rangle=L_{21}(k),
\end{aligned}
$$

which proves that $H(k)$ of eq. (27a) is indeed a real-symmetric matrix. Hence we have to investigate the diagonal matrix elements only. We shall operate with $\left\{C_{2} \mid a_{3} / 2\right\}$ on $L_{11}(k)$ :

$$
\begin{aligned}
L_{11}(k) & =\sum_{n} \exp \left(\mathrm{ik} \cdot R_{n}\right)\left(-\tau / 2|\hat{H}|-\tau / 2+R_{n}\right\rangle=\sum_{n} \exp \left(\mathrm{i} k \cdot R_{n}\right)\left\langle\tau / 2|\hat{H}| \tau / 2+C_{2} R_{n}\right\rangle \\
= & \sum_{n} \exp \left(\mathrm{i} C_{2} k \cdot C_{2} R_{n}\right)\left\langle\tau i 2|\hat{H}| \tau / 2+C_{2} R_{n}\right)=L_{22}\left(C_{2} k\right) .
\end{aligned}
$$

When $k$ is parallel to the $C_{2}$ axis $C_{2} k=k$ so that:

$$
L_{11}(k)=L_{22}(k) \text {. }
$$

When $k$ is perpendicular to $C_{2}$ then $C_{2} k=-k$, but because of inversion symmetry, eq. (29) still holds, since

$$
L_{11}(k)=L_{22}(-k)=L_{22}(k) \text {. }
$$

Alternatively, eq. (29) is satisfied for a general $k$ vector if 


$$
C_{2} R_{n}= \pm R_{n}
$$

A solution for eq. (30) exists only if either $n_{3} \neq 0, n_{2}=0$ and $n_{1}=0$ or $n_{1} \neq 0, n_{2}=0$ and $n_{3}=0$ (and for the trivial case where $n_{1}=0, n_{2}=0$ and $n_{3}=0$, which is of no interest). In other words, eq. (29) is abeyed if all (translationally equivalent) interactions are zero, except those parallel or perpendicular to the rotation axis. In this case, a wavefunction belonging to a general $k$ vector can be represented by eqs. (10) and (II).

\subsection{Four molecules per unit cell}

The site excitation wavefunctions (eqs. (23)-(26)) will be chosen as a basis set and the hamiltonian matrix spanned by this set is given by:

$$
H(k)=\left(\begin{array}{llll}
L_{11}(k) & L_{12}(k) & L_{13}(k) & L_{14}(k) \\
L_{21}(k) & L_{22}(k) & L_{23}(k) & L_{24}(k) \\
L_{31}(k) & L_{32}(k) & L_{33}(k) & L_{34}(k) \\
L_{41}(k) & L_{42}(k) & L_{43}(k) & L_{44}(k)
\end{array}\right),
$$

where

$$
\begin{aligned}
& L_{12}(k)=\exp \left[\mathrm{i} k \cdot\left(\tau_{x}+\tau_{y}\right)\right] \sum_{n} \exp \left(\mathrm{ik} \cdot R_{n}\right)\left\langle 0|\hat{H}| R_{n}+\tau_{x}+\tau_{y}\right\rangle, \\
& L_{13}(k)=\exp \left[\mathrm{i} k \cdot\left(\tau_{y}+\tau_{z}\right)\right] \sum_{n} \exp \left(\mathrm{i} k \cdot R_{n}\right)\left\langle 0|\hat{H}| R_{n}+\tau_{y}+\tau_{z}\right\rangle, \\
& L_{14}(k)=\exp \left[\mathrm{i} k \cdot\left(\tau_{z}+\tau_{x}\right)\right] \sum_{n} \exp \left(\mathrm{ik} \cdot R_{n}\right)\left\langle 0|\hat{H}| R_{n}+\tau_{x}+\tau_{z}\right\rangle, \\
& L_{23}(k)=\exp \left[\mathrm{i} k \cdot\left(\tau_{z}-\tau_{x}\right)\right] \sum_{n} \exp \left(\mathrm{ik} \cdot R_{n}\right)\left\langle\tau_{x}+\tau_{y}|\hat{H}| R_{n}+\tau_{y}+\tau_{z}\right\rangle, \\
& L_{24}(k)=\exp \left[\mathrm{i} k \cdot\left(\tau_{z}-\tau_{y}\right)\right] \sum_{n} \exp \left(\mathrm{i} k \cdot R_{n}\right)\left\langle\tau_{y}+\tau_{x}|\hat{H}| R_{n}+\tau_{z}+\tau_{x}\right\rangle, \\
& L_{34}(k)=\exp \left[\mathrm{i} k \cdot\left(\tau_{x}-\tau_{y}\right)\right] \sum_{n} \exp \left(\mathrm{i} k \cdot R_{n}\right)\left\langle\tau_{y}+\tau_{z}|\hat{H}| R_{n}+\tau_{z}+\tau_{x}\right\rangle, \\
& L_{11}(k)=\sum_{n} \exp \left(\mathrm{i} k \cdot R_{n}\right)\left\langle 0|\hat{H}| R_{n}\right\rangle, \\
& L_{22}(k)=\sum_{n} \exp \left(\mathrm{i} k \cdot R_{n}\right)\left\langle\tau_{x}+\tau_{y}|\hat{H}| R_{n}+\tau_{x}+\tau_{y}\right\rangle, \\
& L_{33}(k)=\sum_{n} \exp \left(\mathrm{i} k \cdot R_{n}\right)\left\langle\tau_{y}+\tau_{z}|\hat{H}| R_{n}+\tau_{y}+\tau_{z}\right\rangle, \\
& L_{44}(k)=\sum_{n} \exp \left(\mathrm{i} k \cdot R_{n}\right)\left\langle\tau_{z}+\tau_{x}|\hat{H}| R_{n}+\tau_{z}+\tau_{x}\right\rangle .
\end{aligned}
$$

The phase factor preceding each of the summation symbols in eqs. (32)-(37) ensures $H(k)$ to be real-symmetric. If $k$ is parallel to one of the symmetry ixes, then it is possible to construct the zero-order wavefunctions in- 
voking symmetry only. 1uese wavefunctions are given in section 3.2 by eqs. (19)-(22). H(k) is diagonalized by a simple real-orthogonal matrix $\mathbf{U}$ :

$$
U=\frac{1}{2}\left(\begin{array}{rrrr}
1 & i & 1 & 1 \\
1 & 1 & -1 & -1 \\
1 & -1 & 1 & -1 \\
1 & -1 & -1 & 1
\end{array}\right) \text {. }
$$

We shall now investigate the effect of various symmetry operators on the matrix elements of $H(k)$ [eqs. (32)-(41)]. Since the hamiltonian is invariant under symmetry operations, only the form of the various matrix elements changes under these operations. Operating with $\left\{c_{x} \mid \tau_{x}+\tau_{y}\right\}$ on $L_{11}(k)$ [eq. (38)], the following change of form is induced:

$$
L_{11}(k)=\sum_{n} \exp \left(\mathrm{i} c_{x} k \cdot R_{n}\right)\left\langle\tau_{x}+\tau_{y}|\hat{H}| R_{n}+\tau_{x}+\tau_{y}\right\rangle=L_{22}\left(c_{x} k\right)
$$

The diagonal elements of $H(k), L_{11}(k)$ and $L_{22}(k)$ are equal if either $c_{x} k=k$ or $c_{x} k=-k$, implying that $k$ is either parallel or perpendicular to the $x$-axis. Similarly, it can be shown that all the diagonal matrix elements are equal if also $c_{y} k= \pm k$ and $c_{z} k= \pm k$. To sum up, if the wave vector lies parallel to one of the symmetry axes, the diagonal matrix elements of $H(k)$ are all equal. Alternatively, all diagonal matrix elements of $H(k)$ are equal if the following relations hold for the translation vector $\boldsymbol{R}_{\boldsymbol{n}}$ :

$$
\begin{aligned}
& c_{x} \boldsymbol{R}_{n}= \pm \boldsymbol{R}_{n}, \\
& c_{y} \boldsymbol{R}_{n}= \pm \boldsymbol{R}_{n}, \\
& c_{z} \boldsymbol{R}_{n}= \pm \boldsymbol{R}_{n} .
\end{aligned}
$$

It is clear from eqs. (44) that these diagonal matrix elements are equal for a general $k$, if interactions between tronslational equivalent molecules are non-zero in the directions of the symmetry axes only, while all other interactions between equivalent molecules are zero.

The situation for the interchange equivalent molecules is somewhat more complicated. Operating with $\left\{c_{x} \mid \tau_{x}+\tau_{y}\right\}$ on eqs. (33) and (34) we arrive at the following expression for the off-diagonal elements:

$$
\begin{aligned}
& L_{13}(k)=L_{24}\left(c_{x} k\right), \\
& L_{14}(k)=L_{23}\left(c_{x} k\right),
\end{aligned}
$$

and operating with $\left\{c_{y} \mid \tau_{y}+\tau_{z}\right\}$ on eq. (32) we obtain:

$$
L_{12}(k)=L_{34}\left(c_{y} k\right) \text {. }
$$

Thus, for $\boldsymbol{k}$ in the direction of the symmetry axes (for any interaction), the $H(k)$ matrix has the following shape:

$$
H(k)=\left(\begin{array}{llll}
\alpha & \delta & \gamma & \beta \\
\delta & \alpha & \beta & \gamma \\
\gamma & \beta & \alpha & \delta \\
\beta & \gamma & \delta & \alpha
\end{array}\right) .
$$

This matrix is diagonalized by the orthogonal matrix, eq. (42). It will be shown that under certain restrictions on the interchange equivalent interactions, the matrix $H(k)$ for a general $k$ has the shape of eq. (46). Since this is a 
more complicated case than the previous cases of translationally equivalent interactions, a detailed derivation is given. The set of symmetry operations, $\hat{O}_{n}$ will be applied to eq. (34), where $\dot{\theta}_{n}$ is given by:

$$
\hat{O}_{n}=\hat{O}\left(\left\{c_{y} \mid \tau_{y}+\tau_{z}+2 \tau_{x}-c_{y} R_{n}\right\}\right) \text {. }
$$

We shall replace the crystal hamiltonian operator $\hat{H}$ in the individual terms of the summation in eq. (34) by the corresponding operators $\hat{O}_{n}^{\dagger} \hat{H} \hat{O}_{n}$ :

$$
\begin{aligned}
L_{14}(k) & =\exp \left[\mathrm{ik} \cdot\left(\tau_{z}+\tau_{x}\right)\right] \sum_{n} \exp \left(\mathrm{i} k \cdot R_{n}\right)\left\langle\hat{O}_{n}[0]|\hat{H}| \hat{o}_{n}\left[R_{n}+\tau_{x}+\tau_{z}\right]\right\rangle \\
& =\exp \left[\mathrm{i} k \cdot\left(\tau_{z}+\tau_{x}\right)\right] \sum_{n} \exp \left(\mathrm{i} k \cdot R_{n}\right)\left\langle\tau_{y}+\tau_{z}+2 \tau_{x}-c_{y} R_{n}|\hat{H}| \tau_{x}+\tau_{z}\right\rangle \\
& =\exp \left[\mathrm{i} k \cdot\left(\tau_{z}-\tau_{x}\right)\right] \sum_{n} \exp \left(\mathrm{i} k \cdot\left(R_{n}+2 \tau_{x}\right)\right]\left\langle\tau_{x}+\tau_{z}|\hat{H}| \tau_{y}+\tau_{z}-c_{y}\left(R_{n}+2 \tau_{x}\right)\right\rangle \\
& =\exp \left[\mathrm{i} k \cdot\left(\tau_{z}-\tau_{x}\right)\right] \sum_{n} \exp \left(\mathrm{i} k \cdot R_{n}\right)\left\langle\tau_{x}+\tau_{z}|\hat{H}| \tau_{y}+\tau_{z}-c_{y} R_{n}\right\rangle .
\end{aligned}
$$

By comparing eq. (48) with eq. (35), we observe that $L_{14}(k)$ is equal to $L_{23}(k)$ if the following relation holds for $\boldsymbol{R}_{n}$ :

$$
\boldsymbol{R}_{n}=-c_{y} \boldsymbol{R}_{n}
$$

Eq. (49) implies that the equality $L_{14}(k)=L_{23}(k)$ can be achieved for a general $k$ vector, if non-zero interactions between interchange equivalent molecules, appearing in eqs. (34) and (35), are confined to planes parallel to the $x z$ plane only. Similar results can be derived for the other off-diagonal matrix elements of $H(k)$. Thus, if all nonzero interactions between interchange equivalent elements are confined to planes parallel to the $x y, y z, z x$ planes we have: $L_{12}(k)=L_{34}(k), L_{13}(k)=L_{24}(k)$ and $L_{14}(k)=L_{23}(k)$, for a general $k$ wavevector.

To conclude, $H(k)$ has the form of matrix eq. (46) if non-zero interactions between translational pairs of equivalent molecules lie parallel to a rotation axis, while non-zero interactions between pairs of intercharge equivalent molecules lie parallel to the symmetry planes.

\section{Appendix. The determination of the trace of the reducible representation for an inversion operation}

Operating on the localized excitation $\left|\tau / 2+R_{n}\right\rangle$ by the symmetry operator $\hat{O}\left\{I\left|\tau+R_{n}\right\rangle\right.$ we obtain for an ungerade function:

$$
\hat{O}\left\{I \mid R_{n}+\tau\right\}\left|\tau / 2+R_{m}\right\rangle=-\left|\tau / 2-R_{m}+R_{n}\right\rangle .
$$

The condition for which eq. (A1) furnishes a non-zero contribution to the trace of the reducible representation $\Gamma$ is given by:

$$
2 R_{m}=R_{n} \text {. }
$$

Because of the cyclic boundary condition eq. (A2) does not have a unique solution. The solution of (A2) can be derived from the simultaneous congruent equations:

$$
n_{1}=2 m_{1}\left(\bmod N_{1}\right) ; \quad n_{2}=2 m_{2} \quad\left(\bmod N_{2}\right) ; \quad n_{3}=2 m_{3} \quad\left(\bmod N_{3}\right) .
$$

Whenever there is a solution for eq. (A3) there exist eight such solutions implying that eight molecules are invariant under a single inversion operation. This is an unphysical results, but an essential consequence of the cyclic boundary condition. 
In a similar manner, congruent equations can be derived for the operator $\delta\left\{I \mid \tau+R_{n}\right\}$ and the localized excitation $\left(-\boldsymbol{\tau} / 2+\boldsymbol{R}_{n}\right)$. These are:

$$
n_{1}=2 m_{1}+1 \quad\left(\bmod N_{1}\right) ; \quad n_{2}=2 m_{2} \quad\left(\bmod N_{2}\right) ; \quad n_{3}=2 m_{3}+1 \quad\left(\bmod N_{3}\right) .
$$

We have here eight solutions, or none. The results for the trace of $\left\{I \mid R_{n}+\tau\right\}$ as determined from eqs. (A3) and (A4) can be represented in a compact form, and is given by eq. (9) of section 3.

A simple example can illustrate the pertinent results of this discussion. We assume a one-dimensional crystal containing ten unit cells with one molecule per unit cell. The diagonal elements of $\Gamma$ for $\delta\{I \mid 4 a\}$ are given by:

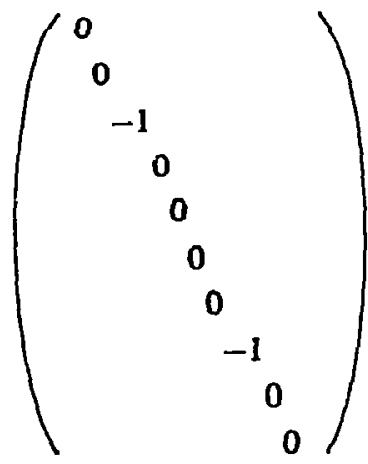

where $a$ is the primitive lattice vector.

\section{References}

[1] A.S. Davydov, JETP 18 (1946) 210;

if. Winston and R.S. Halford, J. Chem. Phys. 17 (1949) 607.

[2] A.S. Davydov, Soviet Phys. Uspekhi 82 (1964) 393 [English tri rslation 7 (1964) 145];

R. Kopelman, J. Chem. Phys. 47 (1967) 2631.

[3] E.I. Rashba, Sov. Phys. Sol. State 5 (1963) 1040 [English translatio. 5 (1963) 757];

S.D. Colson, R. Kopeiman and G.W. Robinson 47 (1967) 27, 5462.

[4] S.D. Colson, D.M. Hanson, R. Kopelman and G.W. Robinson, J. Chem. Phys. 48 (1968) 2215.

[5] E.I. Rashba, Opt. Spectry (USSR) English Transl. 2 (1957) 568;

D.P. Craig and M.R. Philpott, Proc. Roy. Soc. A290 (1966) 583, 602; A293 (1966) 213;

B. Sommer and J. Jortner, J. Chem. Phys. $S 0$ (1969) 187, 822, 839;

D.M. Hanson, R. Kopelman and G.W. Robinson, J. Chem. Phys. 51 (1969) 212.

[6] J. Hoshen and R. Kopelman, to be published.

[7] E. Wigner, Group Theory (Academic Press, New York, 1959).

[8] M. Hamermesh, Group Theory and its Application to Physical Problems (Addison-Wesley, Reading, 1962).

[9] O.U. Kovalev. Irreducible Representations of the Space Groups (Grodon and Breach, New York, 1965).

[10] G. Birkhorf and S. MacLane, A Survey of Modern Algebra (MacMillan, New York, 1965). 